\title{
VARIABLE DENSITY COMPRESSED IMAGE SAMPLING
}

\author{
Zhongmin Wang ${ }^{\dagger}$, Gonzalo R. Arce $e^{\dagger}$ and Jose L. Paredes ${ }^{\star}$ \\ $\dagger$ Dep. of Electrical and Computer Engineering, \\ University of Delaware, Newark, DE, 19716 USA. \\ e-mail:\{zhongmin,arce\}@ee.udel.edu \\ ${ }^{\star}$ Electrical Engineering Department, \\ Universidad de Los Andes, Venezuela \\ e-mail:paredesj@ula.ve
}

\begin{abstract}
Compressed sensing (CS) provides an efficient way to acquire and reconstruct natural images from a reduced number of linear projection measurements at sub-Nyquist sampling rates. A key to the success of CS is the design of the measurement ensemble. This paper addresses the design of a novel variable density sampling strategy, where the "a priori" information about the statistical distributions that natural images exhibit in the wavelet domain is exploited. Compared to the current sampling schemes for compressed image sampling, the proposed variable density sampling has the following advantages: 1) The number of necessary measurements for image reconstruction is reduced; 2) The proposed sampling approach can be applied to several transform domains leading to simple implementations. In particular, the proposed method is applied to the compressed sampling in the 2D ordered discrete Hadamard transform (DHT) domain for spatial domain imaging. Furthermore, to evaluate the incoherence of different sampling schemes, a new metric that incorporates the "a priori" information is also introduced. Extensive simulations show the effectiveness of the proposed sampling methods.
\end{abstract}

\section{INTRODUCTION}

By exploiting the sparsity of natural images, compressed sensing has shown that it is feasible to acquire and reconstruct natural images from a limited number of linear projection measurements at sub-Nyquist sampling rates $[1,2]$. A key component to the success of $\mathrm{CS}$ is the design of the measurement ensemble, which is based on the evaluation of the incoherence between the measurement ensemble and the sparsity basis. Incoherence property requires that the components of the measurement ensemble should have a dense representation in the sparsity basis. Due to the large scale of natural images and their high resolution, it is also required that the generation of the measurement ensemble be both computationally efficient and memory efficient. Furthermore, the sampling scheme should enable fast algorithms to perform image reconstruction.

Measurement matrices where each entry is an independent and identically distributed (i. i. d.) random variable obeying Gaussian or Bernoulli distribution have been proposed for compressed image sampling [1,2]. Recently, it has been shown that the performance of CS sampling can be improved if the random measurement matrices are suitably optimized [3]. Due to their unstructured nature, however, large memory space and demanding computation resources are needed, making them prohibitively expensive for a practical implementation. A more desirable way to obtain linear measurements is by incoherent sampling in a transform domain equipped with fast transform algorithms [4]. Measurement ensembles in the transform domain that enable fast computations include partial Fourier ensemble, scrambled Fourier ensemble (SFE), scrambled block Hadamard ensemble (SBHE) and Noiselets [1, 4, 5]. Although these sampling approaches have shown to obtain good performance in CS applications, their formulation do not exploit any "a priori" information of natural images that could lead to improved CS performance $[6,7]$.

In this paper, we propose a new method for compressed image sampling by exploiting the "a priori" information about the statistical distributions that natural images exhibit in the wavelet domain. A novel family of variable density sampling patterns are designed for compressed image sampling in the frequency transform domains which includes Discreet Fourier Transform (DFT), Discrete Cosine Transform (DCT) and ordered DHT. Equipped with fast transform algorithms, the sampling process is simple, fast and easily implemented. Compared with other sampling strategies, the CS performance obtained with the proposed sampling method is significantly improved.

To design the proposed measurement matrices, a simplified model to describe the statistical distribution of image wavelet coefficients is first established. According to the subband incoherent sampling method proposed in [4], the Fourier coefficients in the subband where significant energy of the wavelet exits should be sampled randomly to minimize the coherence between the sparse wavelets and the measurement Fourier atoms. Thus, based on subband incoherent sampling, we derive a variable density sampling function $p(m, n)$ in the frequency transform domains according to the statistical wavelet model. Here $p(m, n)$ indicates the probability that the $(m, n)$ th coefficient is sampled. The sampling pattern in the frequency transform domain is generated randomly from the proposed density sampling function, where samples are generated according to the underlying probability function.

Compared with other sampling patterns, such as radial sampling pattern or variable density spiral that also exploit some " $a$ priori" information about the image of interest $[1,6]$, the proposed sampling patterns are not heuristically constructed, but are based on reliable statistical models of wavelet coefficients and thus the proposed sampling patterns are analytically justified. Compared with the method proposed in [8], which samples the signals directly in the wavelet domain, the proposed sampling method is more general and does not rely on any specific wavelet basis.

Image reconstruction from a reduced set of random samples is inevitably exacerbated by the interference resulting from undersampling, which depends on the incoherence of the sampling patterns. To compare different sampling patterns, an effective metric to quantitatively evaluate the incoherence of the sampling patterns is desirable. The point spread function (PSF) and transformed point spread function (TPSF) has been proposed in [7] to evaluate the sampling pattern incoherence, but they do not consider the "a priori" information of the underlying signals and thus are not suitable for this work. Therefore, a new incoherence metric is proposed in this paper which incorporates the underlying wavelet statistical models. Simulation results show that there is strong consistence between the low value of the proposed metric and the good quality of the reconstructed image.

\section{COMPRESSED SENSING IN A TRANSFORM DOMAIN}

A signal $\mathrm{x} \in \mathscr{R}^{N}$ is $S$ sparse on some basis $\Psi=\left[\underline{\psi}_{1}, \underline{\psi}_{2}, \ldots, \underline{\psi}_{N}\right]$ if $\mathrm{x}$ can be represented by a linear combination of $S$ vectors from $\Psi$ with $S \ll N$. Thus, the signal can be expressed as: $\mathbf{x}=\Psi \underline{\theta}$, where $\underline{\theta}$ is an $N \times 1$ vector with only $S$ non-zero entries. Compressive signals, such as natural images, can be well approximated by this sparse signal model.

For the application at hand, we consider compressed image sampling in a transform domain $\Phi$. To obtain the sparse signal information, we acquire a small set of transform coefficients of $\mathbf{x}$ in $\Phi$. The measurements are given by: $\mathbf{y}=\Phi_{\Omega} \mathbf{x}$, where $\Phi_{\Omega}$ is a $M \times N$ 
matrix with $M \ll N$ and $\mathbf{y}=\left[y_{1}, y_{2}, \ldots, y_{M}\right]^{T}$ represents the $M$ measurements. Each row of $\Phi_{\Omega}$, denoted by $\phi_{i}$, is taken from a subset $\Omega \subset\{1, \ldots, N\}$ of atoms of $\Phi$. If $\Psi$ and $\Phi$ are incoherent with each other and $\phi_{i}$ is randomly chosen, then given $M=C S \log N \ll N$, where $C \geq \underline{i}$ i is an oversampling factor, $\mathrm{x}$ can be recovered from $M$ measurements with high probability $[1,2]$. The incoherence property holds for many pairs of bases $(\Psi, \Phi)$. If noise is present, the measurements can be modeled as: $\mathbf{y}_{n}=\Phi_{\Omega} \Psi \underline{\theta}+\mathbf{n}$, where $\mathbf{n}$ is zero-mean additive white Gaussian noise. The reconstructed signal can be obtained by using Basis Pursuit Denoising algorithm (BPDN) which solves the following optimization problem [9]:

$$
\min _{\underline{\theta}}\left\|\Phi_{\Omega} \Psi \underline{\theta}-\mathbf{y}_{n}\right\|_{2}^{2}+\lambda\|\underline{\theta}\|_{1},
$$

where $\|\theta\|_{1}=\sum_{i}\left|\theta_{i}\right|, \mathbf{y}_{n}=\Phi_{\Omega} \Psi \theta+\mathbf{n}$, and $\lambda>0$ depends on the noise level. Note that BPDN works equally well for the approximate reconstruction of compressible signals [9]. If there exist fast algorithms associated with both $\Phi$ and $\Psi$, then a fast reconstruction algorithm can be implemented for signal reconstruction [10]. A second image reconstruction algorithm widely used is the minimization of total variation (min-TV) with quadratic constraints. Let $\mathbf{x}(i, j)$ be a $N \times N$ image, then min-TV with quadratic constraints aims to solve the following problem [11]:

$$
\min _{\mathbf{x}}\|\mathbf{x}\|_{T V} \quad \text { s. t. }\left\|\Phi_{\Omega} \mathbf{x}-\mathbf{y}_{n}\right\| \leq \varepsilon
$$

where $\|\mathbf{x}\|_{T V}=\sum_{i, j} \sqrt{[x(i+1, j)-x(i, j)]^{2}+[x(i, j+1)-x(i, j)]^{2}}$ is the total variation and $\varepsilon>0$ is a constant depending on the noise level. Min-TV with quadratic constraints usually provides better quality of image reconstruction at the cost of more computation than BPDN.

\section{VARIABLE DENSITY SAMPLING IN THE FOURIER DOMAIN}

Wavelets have well defined spectral characteristics in the Fourier domain [12]. A coarse scale wavelet has its spectrum localized in the low frequency band whereas a fine scale wavelet has its spectrum widely spread out in the high frequency band. In [4], Fourier sampling of wavelet subbands is proposed to acquire signals sparse in the wavelet domain. To minimize the coherence between the sparse wavelets and the selected Fourier atoms, the Fourier atoms are chosen in the region where significant energy in the wavelet spectrum exists. Let $k=1,2, \ldots, K$ denote the scale of a 1D wavelets where $k=1$ is the finest scale and $k=K$ is the coarsest scale. Without loss of generality, it is assumed that the 1D wavelet has length $N=2^{K}$. Let $\varphi_{k, l}$ denote the $1 \mathrm{D}$ wavelet at a scale $k$ with a shift $l \in\left[0, N 2^{-k}-1\right]$, then the discrete Fourier transform (DFT) spectrum of $\varphi_{k, l}$ is approximately over the band $\mathscr{B}_{k}=\left[-N 2^{-k},-N 2^{-k-1}\right] \cup\left[N 2^{-k-1}, N 2^{-k}\right]$. To reconstruct $\varphi_{k, l}$ from its DFT samples, we need to select the DFT atoms randomly within the band $\mathscr{B}_{k}$ [4]. The probability that a DFT atom within $\mathscr{B}_{k}$ will be sampled depends on the size of $\mathscr{B}_{k}$ and on the number of significant wavelets over $\mathscr{B}_{k}$. The sampling strategy can be naturally extended to $2 \mathrm{D}$ image sampling.

Assuming a natural image of size $N \times N$, we establish a statistical model to describe the distribution of the image in the $2 \mathrm{D}$ wavelet domain. For mathematical tractability, we assume that the statistical distribution of 2D wavelet coefficients at each subband can be modeled as Gaussian distributions with variance at each scale decreasing exponentially from coarse scales to fine scales $[13,14]$. Let $w_{i, j, k}^{B}$ denotes the $(i, j)$ th wavelet coefficient in the $k$ th scale of the subband $B \in L H, H L, H H$. It is assumed that $w_{i, j, k}^{B}$ obey i. i. d. zero-mean Gaussian distribution. That is: $w_{i, j, k}^{B} \sim \mathscr{N}\left(0, \sigma_{k}^{2}\right)$. The variance $\sigma_{k}^{2}$ decreases exponentially from coarse scales to fine scales [14]:

$$
\sigma_{k}^{2}=2^{-a(K-k)},
$$

where $a>0$. It was shown in [14] that $a$ can range from 2.25 to 3.1 based on the inference from empirical studies. The coarsest scaling coefficient of the natural image belongs to a uniform distribution $w_{0} \sim U(0,1)$. The model used in this work is simplified where correlations between the wavelet coefficients are not accounted for. Although more accurate statistical models for wavelet coefficients exist, this model is sufficiently accurate for sampling purposes and allows us to pursue further analysis. $[14,15]$.

Assume that the most significant wavelet coefficients are those whose magnitudes are larger than a given threshold $\mu>0$. Since the number of wavelet coefficients at scale $k<K$ is $3\left(4^{K-k}\right)$, it can be shown that the mean of the number of significant wavelet coefficients at scale $k$ is approximated as:

$$
\lambda_{k} \approx 3\left(4^{K-k}\right) \frac{2}{\sigma_{k} \sqrt{2 \pi}} \int_{\mu}^{\infty} \mathrm{e}^{-\frac{x^{2}}{2 \sigma_{k}^{2}}} d x=3\left(4^{K-k}\right)\left[1-\operatorname{erf}\left(\frac{\mu}{\sqrt{2} \sigma_{k}}\right)\right],
$$

where $\operatorname{erf}(x) \triangleq \frac{2}{\sqrt{\pi}} \int_{0}^{x} e^{-t^{2}} d t$. Furthermore, let $s=\max \{|m|,|n|\}$ where $-N / 2<m, n<N / 2$. Then the $(m, n)$ th 2D DFT atom lies within the spectrum $\mathscr{B}_{k}$ corresponding to the wavelet scale $k=K-\left\lfloor\log _{2}(2 s)\right\rfloor$. It is clear that the number of DFT atoms within $\mathscr{B}_{k}$ is approximately $\varsigma_{k} \approx 3\left(4^{K-k}\right)$. Thus, based on the subband incoherent principle, the probability that the $(m, n)$ th DFT atom is selected should satisfy:

$$
\tilde{p}_{k}(m, n) \propto \frac{\lambda_{k}}{\varsigma_{k}} \approx 1-\operatorname{erf}\left(\frac{\mu}{\sqrt{2} \sigma_{k}}\right) .
$$

We are interested in the asymptotic behavior of $\tilde{p}_{k}(m, n)$ when $k \rightarrow$ 1 . Note that in that case, $\sigma_{k} \ll 1$ and thus $\frac{\mu}{\sqrt{2} \sigma_{k}} \gg 1$. Then, using the fact that $\operatorname{erf}(x) \approx 1-\frac{e^{-x^{2}}}{x \sqrt{\pi}}$, for $x \gg 1, \tilde{p}_{k}(m, n)$ can be approximated as:

$$
\tilde{p}_{k}(m, n) \propto \frac{\sigma_{k}}{\mu} e^{-\frac{\mu^{2}}{2 \sigma_{k}^{2}}} \approx \frac{(2 s)^{-\frac{a}{2}}}{\mu} e^{-\left(\frac{\mu^{2}}{2^{1-a}} s^{a}\right)}
$$

In Eq. (5), the term $e^{-\left(\frac{\mu^{2}}{2^{1-a}} s^{a}\right)}$ determines the asymptotic behavior of $\tilde{p}_{k}(m, n)$. Thus, it is clear that the decreasing rate of $\tilde{p}_{k}(m, n)$ alone with the atom coordinate $s$ depends on the the term $e^{-\left(\frac{\mu^{2}}{2^{1-a}} s^{a}\right)}$. For the random selection of DFT atoms, it is convenient to construct a sampling density function in the DFT domain and generate a sampling pattern according to the sampling density function.

To conform to the decaying behavior of the sampling probability with increasing coordinates while keeping a simple form of the sampling function, we propose a new family of sampling density functions containing only exponential terms. Assuming the size of the underlying image is $M \times N$, the probability that the $(m, n)$ th coefficient is sampled reduces to:

$$
p_{F}(m, n)=e^{-\frac{\left(\sqrt{\left(\frac{m}{M}\right)^{2}+\left(\frac{n}{N}\right)^{2}}\right)^{a_{F}}}{\sigma_{F}^{2}}},
$$

where $-M / 2 \leq m<M / 2,-N / 2 \leq n<N / 2$. $a_{F}$ depends on the characteristics of the underlying image and is directly related to $a$ in (3). From Eq. (5), it is clear that $a_{F}$ should be slightly larger than $a$ to capture the decaying behavior of the sampling probability. If an estimation of $a$ is not available, then setting $a_{F}=3.5$ is recommended since this value leads to robust and satisfactory CS performance. $\sigma_{F}$ is a positive parameter which is tuned to obtain desired number of samples.

The sampling patterns generated from the sampling density function are binary where 1 at $(m, n)$ indicates a sampling point and 0 means no measurement on that point is made. With a probability given by $p_{F}(m, n), 1$ will be generated at $(m, n)$; otherwise, 0 will be generated at $(m, n)$. The local density of the samples approximates the value of the sampling density function and it can be shown that the number of samples generated is monotonically increasing with $\sigma_{F}$. Thus, it is easy to tune the parameters $\sigma_{F}$ iteratively to get the desired number of samples. 


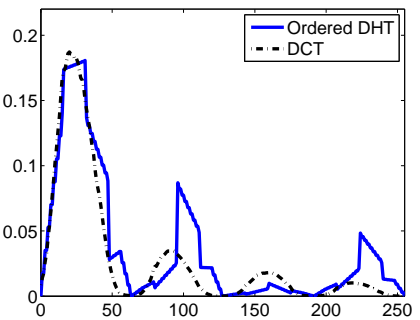

(a)

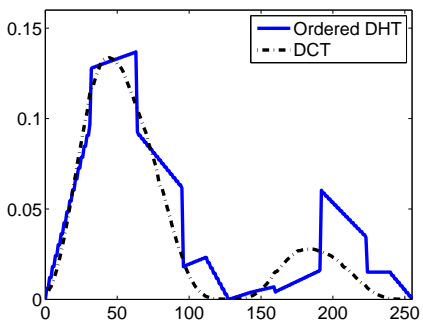

(b)

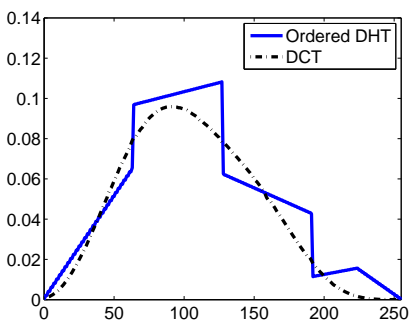

(c)

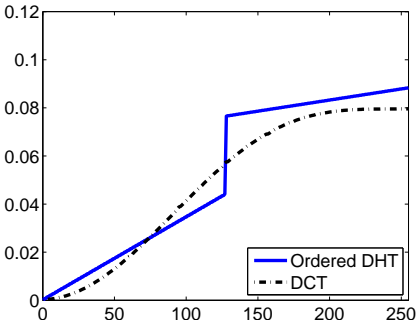

(d)

Figure 1: Ordered DHT and DCT spectra of Daubechies-4 wavelets (a) at scale 4; (b) at scale 3; (c) at scale 2; (d) at scale 1.

\section{VARIABLE DENSITY SAMPLING IN THE ORDERED DHT DOMAIN}

Sampling in the ordered DHT domain is suitable for image sampling with hardware capable of representing binary measurement matrices. An appealing feature of ordered DHT is that the 2D basis images are binary and can be easily implemented by digital micro-mirror devices (DMD) or liquid crystal spatial light modulators (LC-SLM) [16]. Furthermore, ordered DHT is equipped with fast transform algorithms. In ordered DHT, the atoms are ordered by the number of sign changes (zero crossing) between consecutive entries in a Hadamard atom [17]. Thus, ordered DHT can be regarded as a generalized class of DFT and more specifically, a binarized version of DCT sharing thus many properties of DFT and DCT [18].

To design the incoherent sampling pattern in the ordered DHT domain, we exploit the fact that ordered DHT can be thought of as a binary approximation of the DCT. To this end, we first describe the spectrum characteristics of the 1D wavelets in the DCT domain. Let $v_{k, l}(m), 0 \leq m \leq N-1$ be the the DCT of a wavelet $\varphi_{k, l}(n)$, $n=0,1, \ldots, N-1$. Define the averaged DCT spectrum of a wavelets at scale $k$ as:

$$
V(m)_{k}=\sum_{l=0}^{N 2^{-k}-1} \operatorname{abs}\left(v_{k, l}(m)\right) .
$$

It can be shown that the DCT spectrum of wavelets at scale $k$ has approximately the same shape as their Fourier spectra [19]. The DCT spectrum band $\mathscr{B}_{k}$ of wavelets at scale $k$ is $\mathscr{B}_{k} \approx\left[N 2^{-k}, N 2^{-k+1}\right]$ [19]. Much like the incoherent sampling in the Fourier domain, to reconstruct $\varphi_{k, l}$ from its DCT samples, we need to select the DCT atoms randomly within the band $\mathscr{B}_{k}$. Now consider the incoherent sampling in the ordered DHT domain. We can define the averaged ordered DHT spectrum of wavelets at scale $k$ as:

$$
H(m)_{k}=\sum_{l=0}^{N 2^{-k}-1} \operatorname{abs}\left(h_{k, l}(m)\right),
$$

where $h_{k, l}(m), 0 \leq m \leq N-1$ is the ordered DHT of $\varphi_{k, l}(n)$. Since ordered DHT is the binary approximation of the DCT, $H(m)_{k}$ can be approximated as: $H(m)_{k} \approx V(m)_{k}$. To further illustrate this point, Fig. 1 shows $H(m)_{k}$ for $k=1$ to 4 where $N=256$ and Daubechies4 wavelets are used. The corresponding DCT spectrum at $k$ scale is also shown. It can be seen that the averaged ordered DHT spectrum and the averaged DCT spectrum are similar, which indicates that the principles of sampling in the DCT domain should be equally applied to the sampling in the ordered DHT domain. Such conclusion also hold for 2D DCT and 2D ordered DHT. Following a similar procedure to that described in Sec. 3, the variable density sampling function in 2D ordered DHT domain is designed as follows:

$$
p_{H}(m, n)=e^{-\frac{\left(\sqrt{\left(\frac{m}{M}\right)^{2}+\left(\frac{n}{N}\right)^{2}}\right)^{a_{H}}}{\sigma_{H}^{2}}},
$$

where $0 \leq m \leq M-1,0 \leq n \leq N-1 . a_{H}$ depends on the parameter $a$ in the image statistical model (3) and $\sigma_{H}>0$ depends on the number of samples. The sampling patterns are then obtained as realizations of the statistical model $p_{H}(m, n)$.

\section{EVALUATION OF INCOHERENCE SAMPLING}

Undersampling in the transform domain inevitably brings aliasing interference in signal reconstruction. However, if the sampling is incoherent, the aliasing interference has noise-like effect and can can be removed by compressed sensing without degrading the image quality [7]. To evaluate the incoherent sampling patterns for natural images we consider the interference in the wavelet domain resulted from undersampling in the measurement transform domain $\Phi$. To this end, we extend the TPSF analysis in [7] and incorporate the statistical model of signal distribution in the wavelet domain.

Let $w_{i, j},(i, j) \in[0, N-1] \times[0, N-1]$, be the simplified notation of the $(i, j)$ th $2 \mathrm{D}$ wavelet coefficient of a natural image that obeys the proposed model in Sec. 3. Incoherence is measured by the interference in the wavelet domain caused by the undersampling in $\Phi$. Without loss of generality, let $\Phi_{u}$ be the undersampled ordered DHT operator and $\Phi_{u}^{+}$be the back-projection ordered DHT operator. Let $\Psi$ be the sparse wavelet transform and $\Psi^{i, j}$ be the $(i, j)$ th wavelet atom. For an image of size $N \times N$, we define a weighted TPSF as: $T_{w}^{i, j}=\left|w_{i, j}\right| \Psi \Phi_{u}^{+} \Phi_{u} \Psi^{i, j} . T_{w}^{i, j}$ is the interference in the wavelet domain caused by the undersampling of $\left|w_{i, j}\right| \Psi^{i, j}$ in ordered DHT. $T_{w}^{i, j}$ is the distribution of the energy of $\left|w_{i, j}\right| \Psi^{i, j}$ to other wavelets through back-projeciton. A nonzero value of $T_{w}^{i, j}$ at $(m, n) \neq(i, j)$ means that the wavelet component at $(m, n)$ suffers from the interference caused by the undersampling of the wavelet component at $(i, j)$. Note that if test image is available, $w_{i, j}$ can also be drawn from the test image directly. The incoherence of the sampling pattern can be evaluated by the following metric $\xi$ defined as:

$$
\xi=\frac{1}{N^{2} \times N^{2}} \sum_{(i, j)} \sum_{(m, n)}\left|\tilde{T}_{w}^{i, j}(m, n)\right|^{2}
$$

where $\tilde{T}_{w}^{i, j}(m, n)$ is defined as: $\tilde{T}_{w}^{i, j}(m, n)=T_{w}^{i, j}(m, n)$ for $(m, n) \neq$ $(i, j)$ and $\tilde{T}_{w}^{i, j}(m, n)=0$ for $(m, n)=(i, j)$. $\xi$ can effectively measure the strength of the aliasing interference. The smaller the $\xi$, the smaller the incoherent interference. As will be shown in the simulations, among several sampling patterns, $\xi$ can be used to select the sampling pattern that yields the lowest incoherence interference.

\section{SIMULATIONS}

Due to the space constraints, only compressed image sampling in the 2D ordered DHT domain is illustrated. The proposed sampling method is applied to acquisition and reconstruction of the natural image "Boat" shown in Fig. 2(a) with size $256 \times 256$. The test image is assumed sparse in the Daubechies- 8 wavelet domain and the pixel values are scaled within interval $[0,1]$. It is also assumed that each measurement is corrupted by additive white Gaussian noise with variance $\sigma^{2}=1 e^{-4}$. The BPDN algorithm and min-TV with quadratic constraints algorithm are used for image reconstruction. 


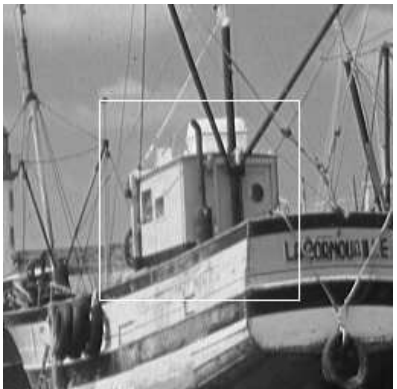

(a)

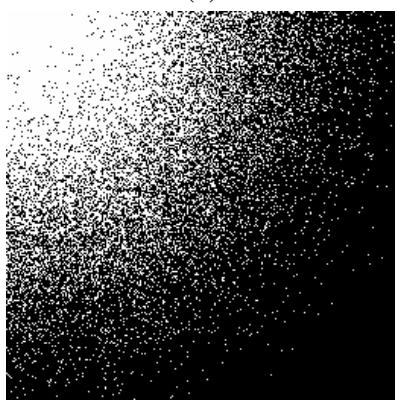

(c)

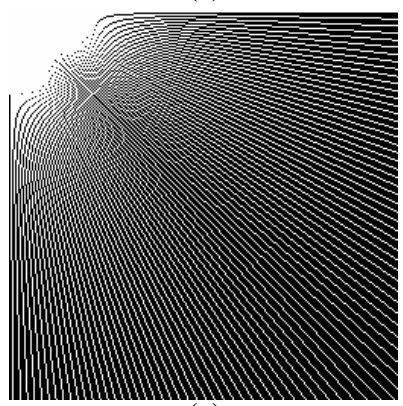

(e)

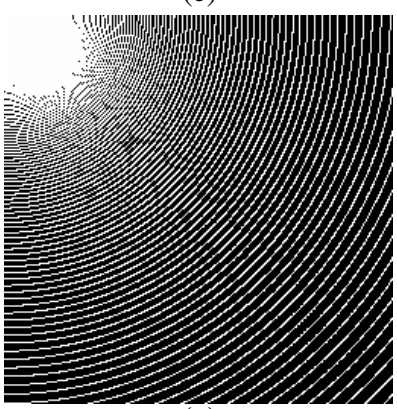

(g)

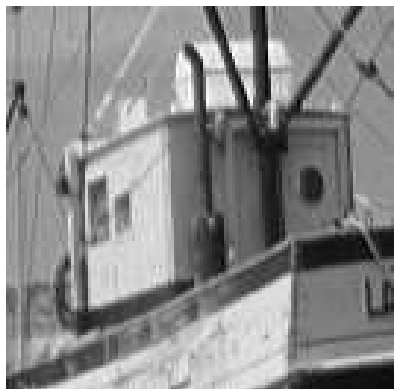

(b)

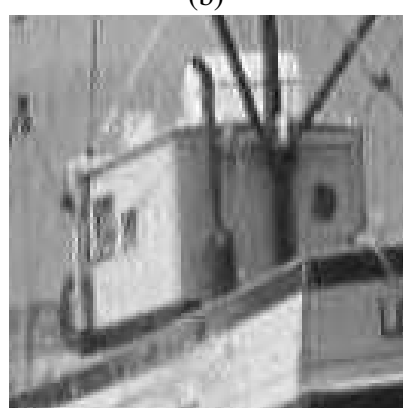

(d)

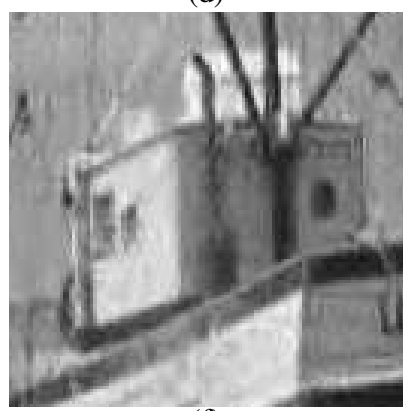

(f)

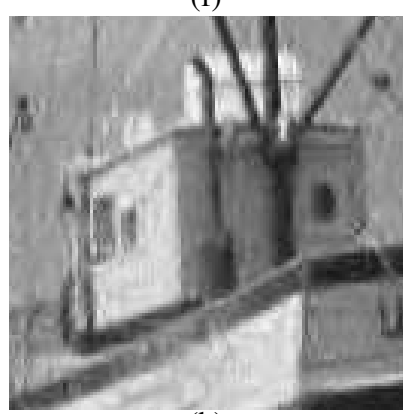

(h)
Figure 2: (a) The original "boat" image. (b) Part of the original image. (c) Proposed variable density sampling pattern. (d) Part of the reconstructed image from the proposed sampling pattern. (e) Radial sampling pattern. (f) Part of the reconstructed image from the radial sampling pattern. (g) Logarithmic spiral sampling pattern. (h) Part of the reconstructed image from the Logarithmic spiral sampling pattern.

Figure 2(c) depicts a realization of the proposed variable density sampling pattern in the ordered DHT domain that contains 20000 sampling points generated from Eq. (9) with $a_{H}=3.5$ and $\sigma_{H}=0.501$. To illustrate more details of the reconstructed image, we compare part of the original image and part of the reconstructed image corresponding to the region within the white frame in Fig. 2(a). The framed area of the original image and the framed area of the reconstructed image are shown in Fig. 2(b) and Fig. 2(d), respectively. It is clear that with an undersampling ratio of $30.5 \%$, the test image is reconstructed with only small distortion.
Table 1: Reconstruction of images in ordered DHT domain. The performance is measured by PSNR (dB). "P. VD" is the proposed variable density sampling.

\begin{tabular}{ccccccc}
\hline No. & P. VD & Radial & Log-spiral & Noiselet & SFE & SBHE \\
\hline \hline & & & boat & & & \\
5000 & $\mathbf{2 2 . 7 5}$ & 19.88 & 19.79 & 18.51 & 19.27 & 18.62 \\
10000 & $\mathbf{2 4 . 9 3}$ & 22.93 & 22.77 & 20.78 & 21.39 & 20.74 \\
15000 & $\mathbf{2 6 . 7 0}$ & 25.09 & 25.03 & 22.67 & 23.25 & 22.69 \\
20000 & $\mathbf{2 8 . 3 6}$ & 26.96 & 26.95 & 24.19 & 24.93 & 24.56 \\
25000 & $\mathbf{2 9 . 5 5}$ & 28.79 & 28.64 & 25.58 & 26.27 & 26.18 \\
\hline \hline & & & Lena & & & \\
5000 & $\mathbf{2 5 . 5 1}$ & 21.28 & 21.51 & 20.14 & 21.25 & 20.05 \\
10000 & $\mathbf{2 7 . 9 1}$ & 25.33 & 25.49 & 23.10 & 23.87 & 23.10 \\
15000 & $\mathbf{2 9 . 7 7}$ & 28.24 & 28.04 & 25.06 & 25.77 & 25.45 \\
20000 & $\mathbf{3 1 . 2 4}$ & 30.07 & 29.91 & 26.76 & 27.56 & 27.43 \\
25000 & $\mathbf{3 2 . 5 7}$ & 31.82 & 31.54 & 28.48 & 29.17 & 29.01 \\
\hline \hline
\end{tabular}

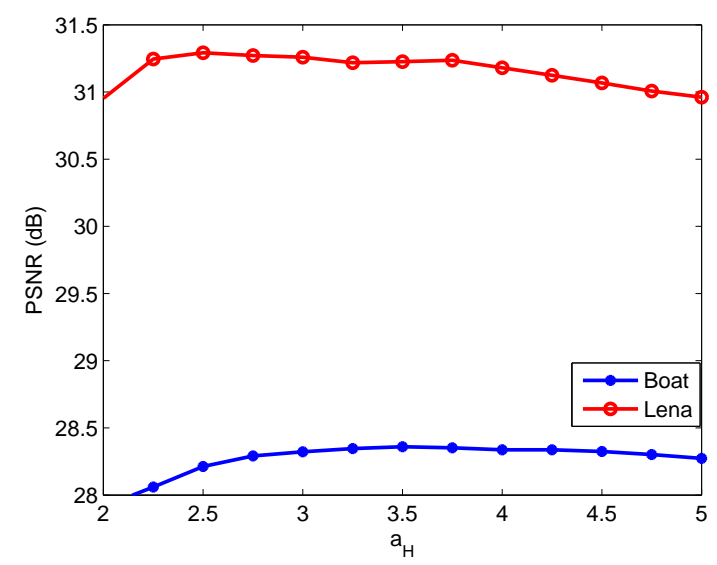

Figure 3: Reconstruction results of image "Boat" and "Lena" from the proposed sampling patterns generated by different $a_{H}$. The number of measurements is 20000 .

For comparison purposes, we also reconstruct the "Boat" image from the samples taken from the radial sampling pattern and logarithmic spiral sampling pattern shown in Fig. 2(e) and Fig. 2(g), respectively. The number of measurements taken from both sampling patterns are the same as that of the proposed sampling pattern. Part of the corresponding reconstructed images are shown in Fig. 2(f) and Fig. 2(h), respectively. Both reconstructed images show more aliasing artifacts, which indicates that exploiting the "a priori" information about the distribution of the wavelet coefficients in the design of the projection operator leads to a better image reconstruction.

More extensive simulations are applied to images boat and Lena with size $256 \times 256$. Simulation results are summarized in Table 1, where the number of measurements ranges from 5000 to 25000 and the best results as the average of ten runs is also highlighted. For each test image, it is shown that the proposed variable density sampling achieves much better performance than logarithmic spiral patterns and radial patterns. The performance gain is $2 \sim 4 \mathrm{~dB}$ with 5000 samples and $0.5 \sim 0.8 \mathrm{~dB}$ with 25000 samples. To verify that the proposed sampling pattern, which exploits the a priori information of natural images, achieves better performance than methods that do not exploit the a priori information, the simulation results using the Noiselet ensemble, SFE and SBHE under different number of measurements are also presented in Table 1. To acquire the image information, samples of the Noiselets, SFE and SBHE are taken randomly. The proposed sampling method achieves the best performance in all simulations. Such comparison clearly shows the performance gain achieved by exploiting the $a$ priori information.

The incoherence of the proposed sampling patterns can also be 
evaluated by using the proposed incoherence metric proposed in this paper. We test three sampling patterns in the ordered DHT domain using the image "Boat" and take 5000 measurements. The incoherence metrics are $0.0034,0.0062$ and 0.0078 for the proposed variable density sampling pattern, radial sampling pattern and logarithmic spiral sampling pattern, respectively. The lowest metric value is yielded by the proposed variable density sampling pattern leading to the least aliasing interference, which, in turn, is in accordance with the best reconstruction performance.

Finally, we show that the reconstruction performance is not very sensitive to the parameters $a_{F}$ or $a_{H}$ in the sampling functions. Here, we test the reconstruction performance as the parameter $a_{H}$ changes. Figure 3 shows the reconstruction of images "Boat" and "Lena" with 20000 measurements. Images "Boat" and "Lena" have different curves and textures, thus have different statistical model parameters. The sampling patterns are constructed from sampling density functions with $a_{H}$ ranging from 2 to 5. It is shown in Fig. 3 that the sampling patterns lead to similar performance for both images when $a_{H}$ ranges from 2.5 to 4.5 . For both images, the difference of performance measured by PSNR is within $0.3 \mathrm{~dB}$ with different $a_{H}$. Thus, the image reconstruction is robust to variations over $a_{H}$. Interestingly, note also that the reconstruction performance tends to become worse as $a_{H}$ increases. Since larger $a_{H}$ means that more low frequency samples are taken, the simulation shows that sampling only low frequency components is not a good strategy.

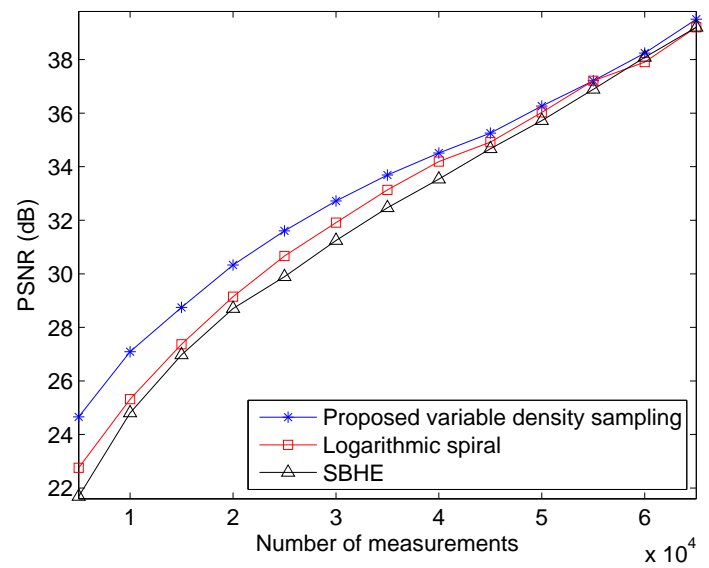

Figure 4: Reconstruction results for image "Boat' using min-TV algorithm with quadratic constraints.

Figure 4 shows the simulation results of image recovery using min-TV. For illustrative purposes, only the following sampling schemes are considered: the proposed variable density sampling, logarithmic spiral sampling and SBHE [5]. The simulation results show that the proposed variable density sampling again achieves the best performance, which is more obvious when the sampling ratio is low. It can be concluded that the advantages of using the proposed variable density sampling does not depend on a specific reconstruction algorithm.

\section{CONCLUSIONS}

In this paper, a family of variable density sampling patterns are proposed for compressed sensing of natural images in the Fourier domain, DCT and ordered DHT domain, which are based on the statistical model of natural images in the sparse wavelet domain. Compared with other sampling scheme, our proposed method is simple, fast and can be extended to a wide range of applications. Furthermore, the "a priori" information needed is pretty general and no data training for parameter estimation is needed. The proposed metric, along with extensive simulation study, show that the proposed sampling pattern leads to the least coherent interference.
[1] E. Candés, J. Romberg, and T. Tao, "Robust uncertainty principles: Exact signal reconstruction from highly incomplete frequency information," IEEE Trans. Inf. Theory, vol. 52, no. 2, pp. 489-509, Feb. 2006.

[2] D. Donoho, "Compressed sensing," IEEE Trans. Inf. Theory, vol. 52, no. 4, pp. 1289-1306, Apr. 2006.

[3] M. Elad, "Optimized projections for compressed sensing," IEEE Trans. Signal Process., vol. 55, no. 12, pp. 5695-5702, Dec. 2007.

[4] E. Candés and J. Romberg, "Sparsity and incoherence in compressive sampling," Inverse Problems, vol. 23, no. 3, pp. 969-985, 2007.

[5] L. Gan, T. T. Do, and T. D. Tran, "Fast compressive imaging using scrambled block Hadamard ensemble," Preprint, 2008.

[6] C. Schróder, P. Bórnert, and B. Aldefeld, "Spatial excitation using variable-density spiral trajectories," $J$ Mag. Reson. Imag., vol. 18, no. 1, pp. 136-141, 2003.

[7] M. Lustig, D. Donoho, and J. M. Pauly, "Sparse MRI: The application of compressed sensing for rapid MR imaging," Mag. Reson. in Med., vol. 58, no. 6, pp. 1182-1195, Dec. 2007.

[8] S. Dekel, "Adaptive compressed image sensing based on wavelet-trees," Preprint, 2008.

[9] S. S. Chen, D. L. Donoho, and M. A. Saunders, "Atomic decomposition by Basis Pursuit," SIAM SCI. Comput., vol. 20, no. 1, pp. 33-61, Aug. 1998.

[10] M. Figueiredo, R. D. Nowak, and S. J. Wright, "Gradient projection for sparse reconstruction: Application to compressed sensing and other inverse problems.," IEEE J. STSP, vol. 1, no. 4, pp. 586-598, Dec. 2007.

[11] E. Candés, J. Romberg, and T. Tao, "Stable signal recovery from incomplete and inaccurate measurements," Comm. Pure Appl. Math, vol. 59, no. 8, pp. 1207-1223, Aug. 2006.

[12] S. Mallat, A Wavelet tour of signal processing, Academic Press, 1998.

[13] V. Strela, "Denoising via block Wiener filtering in wavelet domain," in 3rd Europ. Congress Math. 2000, Birkháuser Verlag.

[14] J. K. Romberg, H. Choi, and R. G. Baraniuk, "Bayesian tree-structured image modeling using wavelet-domain hidden Markov models," IEEE Trans. Image Process., vol. 10, no. 7, pp. 1056-1068, Jul. 2001.

[15] S. Mallat, "A theory for multiresolution signal decomposition: the wavelet representation," IEEE Trans. Pattern Anal. Mach. Intell., vol. 11, no. 7, pp. 674-693, Jul. 1989.

[16] M. Duarte, M. Davenport, D. Takhar, J. Laska, T. Sun, K. Kelly, and R. Baraniuk, "Single-pixel imaging via compressive sampling," IEEE Signal Process. Mag., vol. 25, no. 2, pp. 83-91, Mar. 2008.

[17] Y. Q. Shi and H. Sun, Image and video compression for multimedia engineering, CRC press, 1999.

[18] N. P. Pitsianis, D. J. Brady, A. Portnoy, X. Sun, T. Suleski, M. A. Fiddy, M. R. Feldman, and R. D. Tekolste, "Compressive imaging sensors," in Proc. SPIE, 1999, vol. 6232.

[19] Z. Wang and G. R. Arce, "Variable density compressed image sampling," submitted to IEEE Trans. Image Process.

\section{REFERENCES}

